\begin{tabular}{|c|c|c|c|c|c|c|}
\hline \multirow{4}{*}{ Impact Factor: } & ISRA (India) & $=3.117$ & SIS (USA) & $=0.912$ & ICV (Poland) & $=6.630$ \\
\hline & ISI (Dubai, UAE & $=0.829$ & РИНЦ (Russia & $=0.156$ & PIF (India) & $=1.940$ \\
\hline & GIF (Australia) & $=0.564$ & ESJI (KZ) & $=8.716$ & IBI (India) & $=4.260$ \\
\hline & JIF & $=1.500$ & SJIF (Morocco & $=5.667$ & OAJI (USA) & $=0.350$ \\
\hline
\end{tabular}

\section{SOI: $\underline{1.1 / \text { TAS }}$ DOI: $10.15863 /$ TAS \\ International Scientific Journal \\ Theoretical \& Applied Science}

p-ISSN: 2308-4944 (print) e-ISSN: 2409-0085 (online)

Year: 2019 Issue: $07 \quad$ Volume: 75

Published: $30.07 .2019 \quad$ http://T-Science.org
QR - Issue

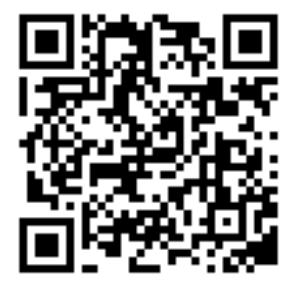

QR - Article

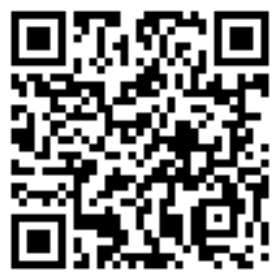

A.A. Qasanov

Azerbaijan State University of Oil and Industry professor, department of petrochemical technology and industrial ecology, faculty of chemical technology,

Azerbaijan, Baku

M. Y. Abdullayeva Azerbaijan State University of Oil and Industry associate professor, department of petrochemical technology and industrial ecology, faculty of chemical technology, Azerbaijan, Baku mayaabdullayeva@hotmail.com

\title{
STUDYING THE PROCESS OF LIQUID EXTRACTION IN A COUNTERCURRENT APPARATUS
}

Abstract: It has been assumed that by the help of the experiments the component quantity extracted during the contact between phases is divided into three parts: at the periods of lifting, falling and coalescence of the drops. Equations for calculation of diffusion coefficients of mass transfer for all three periods have been offered. Coefficients of molecular diffusion, masstransfer and massefficiency have been calculated on the offered methodics.

Key words: extraction, coefficients, massexchange, masstransfer, drop, diameter, circulation, falling.

Language: Russian

Citation: Qasanov, A. A., \& Abdullayeva, M. Y. (2019). Studying the process of liquid extraction in a countercurrent apparatus. ISJ Theoretical \& Applied Science, 07 (75), 376-381.

Soi: http://s-o-i.org/1.1/TAS-07-75-62 Doi: crossef https://dx.doi.org/10.15863/TAS.2019.07.75.62

Classifiers: UDC 66.01.77, Chemistry and chemical technology.

\section{ИЗУЧЕНИЕ ПРОЦЕССА ЖИДКОСТНОЙ ЭКСТРАКЦИИ В ПРОТИВОТОЧНОМ АППАРАТЕ}

Аннотация: При помощзи экспериментов, предположено, что количество компонента экстрагируемого за время контакта между фазами делится на три части, в периодах подъема, падения и коалесиенции капель. Предложены уравнения для расчета коэффициентов диффузии массопередачи для всех трех периодов. Рассчитаны коэффициенты молекулярной диффузии, массопередачи и массоотдачи по предложенной нами методике.

Ключевые слова: экстракичи,, коэффицчиенты, массообмен, массопередача, капля, диаметр, циркулящия, падение.

\section{Введение}

Скорость массопередачи в экстракционных аппаратах зависит от большого числа переменных. Поэтому получение обобщенных расчетных уравнений по массопередаче затруднительно.
Механизм массопередачи, при известной поверхности и времени фазового контакта, исследуют в опытах с единичными каплями. Результаты некоторых исследований приведены в табл. 1. 


\begin{tabular}{|c|c|c|c|c|c|c|}
\hline \multirow{4}{*}{ Impact Factor: } & ISRA (India) & $=3.117$ & SIS (USA) & $=0.912$ & ICV (Poland) & $=6.630$ \\
\hline & ISI (Dubai, UAE & $=0.829$ & РИНЦ (Russia & $=0.156$ & PIF (India) & $=1.940$ \\
\hline & GIF (Australia) & $=0.564$ & ESJI (KZ) & $=8.716$ & IBI (India) & $=4.260$ \\
\hline & JIF & $=1.500$ & SJIF (Morocce & $=5.667$ & OAJI (USA) & $=0.350$ \\
\hline
\end{tabular}

Таблица 1. Кинетика экстракции для единичных капель

\begin{tabular}{|c|c|c|c|}
\hline $\begin{array}{l}\text { Распределяемое } \\
\text { вещество }\end{array}$ & Дисперсная фаза & Сплошная фаза & $\begin{array}{l}\text { Приближенное значение } \\
\text { коэффициента } \\
\text { распределения, } \mathrm{m}^{1}\end{array}$ \\
\hline Бутилгликоль & Изопропиловый эфир & Вода & 0,32 \\
\hline Фенилизопропанол & Изопропиловый эфир & Вода & 0,37 \\
\hline Уксусная кислота & Изопропиловый эфир & Вода & 0,42 \\
\hline
\end{tabular}

Методика определения частных (фазовых) коэффициентов массопередачи, впервые разработанная Кольборном и Уелшем [1,с. 32653281], была применена во многих работах[2,с.3585-3596]. Эта методика заключается в контактировании слаборастворимых жидкостей и в определении скорости насыщения одной фазы в другой.

При помощи экспериментов получены данные, необходимые для определения коэффициентов массопередачи и поверхности контакта фаз в случае единичных капель. Показано, что скорость экстракции, определенная для единичных капель может быть использована при расчете массопередачи в распылительных и сетчатых экстракторах. Таким образом, эффективность промышленных экстракторов этих типов может быть надежно установлена по данным подробного исследования массопередачи для единичных капель.

Общее количество вещества, экстрагируемого за все время контакта между сплошной и диспергируемой фазами, может быть разделено на три части. Вещество в период: образования, падения и коалесценции капель. Кинетические закономерности для каждого из указанных периодов будут рассмотрены ниже.

\section{Материал и методы исследования}

Экстракция в период образования капель. Массопередача в период образования капель изучалась многими исследователями [3.c.471486], которые показали, что из одной фазы в другую может переходить до $20 \%$ от предельно возможного количества распределяемого компонента, соответствующего равновесному. Сходимость значений коэффициентов массопередачи, полученных различными исследователями разные, что объясняется от части трудностями в разработке техники эксперимента.

Наиболее надежным является уравнение для коэффициентов массоотдачи в сплошной фазе

$$
\kappa_{c}=4,6 \sqrt{\frac{D_{\mathrm{M}}}{\pi t_{k}}}
$$

где $\mathrm{t}_{\mathrm{k}}$ - время образования капли;

$D_{\text {м }}$ - коэффициент молекулярной диффузии; $\kappa_{\mathrm{c}}$ - среднее значение коэффициента массоотдачи, расчитанного по конечной величине поверхности капли.

Уравнение (1) аналогично по форме теоретическому уравнению для нестационарной диффузии, но модифицировано с учетом движения жидкости вблизи поверхности раздела. Однако, это уравнение не учитывает ускорение массопередачи за счет осциляции и разрушения капель.

$$
t_{k}=\frac{2 d^{3}}{3 U_{D} D^{2}}
$$

где $d$ - диаметр капли;

$D$ - диаметр эктрактора;

$U_{D}-$ фиктивная скорость дисперсной фазы.

Для единичных капель величина поверхности контакта фаз, используемая совместно с величиной $\kappa_{\mathrm{c}}$ может быть рассчитана по диаметру капли $d$.

Надо отметить, что уравнение (1) применимо для расчета массопередачи только снаружи капли. Во многих случаях величина коэффициента массоотдачи близка к величине коэффициента массопередачи $\kappa_{D}$, так как внутренняя циркуляция, возникающая в капле в период ее образования, обуславливает высокие коэффициенты массоотдачи внутри капли. Однако, если присутствие растворенного вещества в сплошной фазе весьма благоприятно влияет на сдвиг равновесия в сторону этой фазы, то сопротивление массопередачи внутри капель пренебречь нельзя.

Заслуживает внимание тот факт, что для экстракции получены опытные данные по массопередаче, которые могут быть скоррелированы с помощью следующего выражения

$$
\kappa_{D}=0,017 t_{k}^{-0,53}
$$

Это уравнение даст результат, близкий к результату, получаемому по уравнению (1), в том случае, когда коэффициент диффузии колеблется в пределах $0,5 \cdot 10^{-9}-1,6 \cdot 10^{-9} \mathrm{~m}^{2} /$ сек, несмотря на низкое значение коэффициента распределения. Это подтверждает, что внутренняя циркуляция повышает коэффициент массоотдачи внутри капли, который оказывает незначительное влияние на коэффициент массопередачи. К сожалению, для систем, у которых величина m заметна ниже 0,03 , внутреннее сопротивление массопередачи имеет существенное значение, а 


\begin{tabular}{|c|c|c|c|c|c|c|}
\hline \multirow{4}{*}{ Impact Factor: } & ISRA (India) & $=3.117$ & SIS (USA) & $=0.912$ & ICV (Poland) & $=6.630$ \\
\hline & ISI (Dubai, UAE & $=0.829$ & РИНЦ (Russia) & $=0.156$ & PIF (India) & $=1.940$ \\
\hline & GIF (Australia) & $=0.564$ & ESJI (KZ) & $=8.716$ & IBI (India) & $=4.260$ \\
\hline & JIF & $=1.500$ & SJIF (Morocco) & $=5.667$ & OAJI (USA) & $=0.350$ \\
\hline
\end{tabular}

надежных методов для его определения не существует. Нижнее предельное значение $\kappa_{D}$ может быть получено при использовании формулы

$$
\kappa_{D}=3,43 \sqrt{\frac{D_{\mathrm{M}}}{\pi t_{k}}}
$$

Экстракция в период подъема или падения капель. Как и для экстракции, в период образования капель, данные различных исследователей, значительно отличаются. Коэффициенты массопередачи применимы только для той системы, для которой они были определены, и примерно для тех же рабочих условий.

Мы сделали попытку разрешить вопрос на основе теоретических моделей массопередачи для каждой из фаз. Полученные уравнения были сравнены с различными опытными данными и показали удовлетворительную сходимость. Среднеквадратичное отклонение расчетных коэффициентов массопередачи от опытных составляло $30 \%$ при использовании активностей для выражения движущих сил и более $30 \%$ при использовании для той же цели концентраций. Уравнение для коэффициента массопередачи в жидкости, окружающее каплю, т. е. в сплошной фазе $\quad \kappa_{c}=2 \sqrt{\frac{D_{\mathrm{M}} V}{\pi d}}$

где $\mathrm{V}$ - относительная скорость подъема или падения капли, см/сек;

Выражение (5) представляет собой видоизмененное уравнение (1)

$$
\kappa_{\text {ж }}=\frac{2}{\sqrt{\pi}} \sqrt{\frac{D_{\mathrm{M}}}{t_{\mathrm{K}}}}
$$

где $\kappa_{ж}-$ коэффициент массоотдачи в жидкой фазе.

Для использования этого уравнения необходимо знать диаметр капли, который может быть определен с помощью рис. 1.

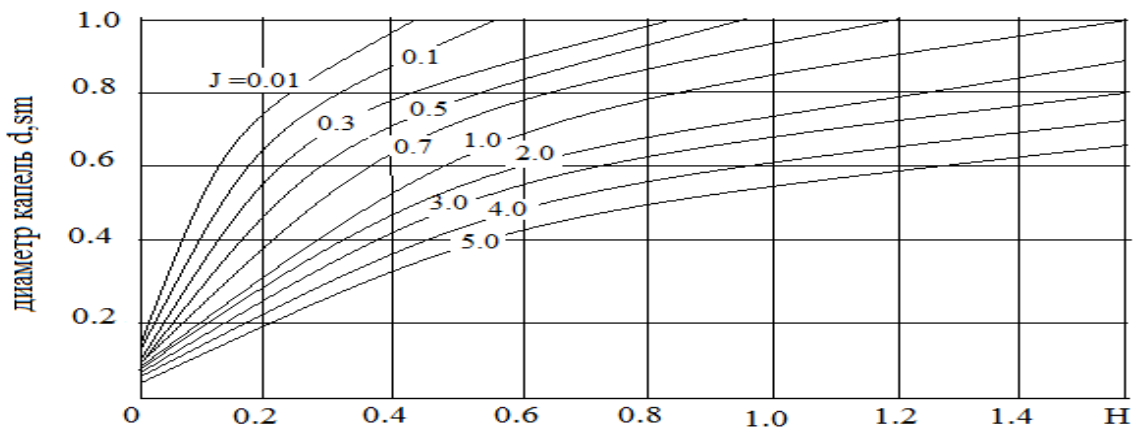

\section{Рис.1. Зависимость диаметра капель, выходящих из отверстий распылительного устройства}

Должна быть также известна скорость движения капли. Для определения ее нужно учитывать является ли диаметр капли больше или меньше некоторой критической величины [4,c.3541].

Критический диаметр капли может быть рассчитан по уравнению

$$
d_{\text {кр }}=0,33 \rho_{c}^{-0,14} \Delta \rho^{-0,43} \mu_{c}^{0,30} \sigma^{0,24}
$$

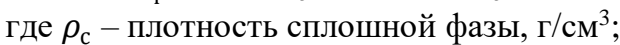

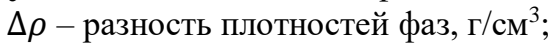

$\mu_{\mathrm{c}}-$ вязкость сплошной фазы, Пз;

$\sigma$ - межфазное натяжение, дин/см.

При $\mathrm{d}<\mathrm{d}_{\text {кр }}$

$$
V_{1}=38,3 \rho_{c}^{-0,45} \Delta \rho^{0,58} \mu_{c}^{-0,11} d^{0,70}
$$

При $\mathrm{d}>\mathrm{d}_{\text {кр }}$

$$
V_{2}=17,6 \rho_{c}^{-0,55} \Delta \rho^{0,28} \mu_{c}^{0,10} \text { (9) }
$$

Уравнения (8), (9) отличаются от уравнений для твердых сфер, тем, что в них учитываются осцилляция капель и отклонения формы капель от шарообразной.
Для расчета коэффициента массоотдачи в диспергируемой фазе

$$
\kappa_{D}=0,00375 \frac{V}{1+\frac{\mu_{D}}{\mu_{C}}}
$$

Уравнение (10) основано на модели, которая исходит из обновления поверхности капель, обусловленного внутренней циркуляцией жидкости в них. В этом уравнении не учитывается влияние межфазного натяжения на внутреннюю циркуляцию в капле, что является наиболее серьезным недостатком модели, опытные величины коэффициентов массоотдачи и значения их, рассчитанные по уравнению (10), по крайней мере на порядок выше значений, получаемых при допущении, что массообмен происходит за счет молекулярной диффузии в шарообразную каплю без внутренней циркуляции в период ее подъема или падения. Уравнения (5) и (10) дают наилучшие результаты применительно к большим каплям, для которых силы межфазового натяжения пренебрежимо малы и низким 


\begin{tabular}{|c|c|c|c|c|c|c|}
\hline \multirow{4}{*}{ Impact Factor: } & ISRA (India) & $=3.117$ & SIS (USA) & $=0.912$ & ICV (Poland) & $=6.630$ \\
\hline & ISI (Dubai, UAE & $=0.829$ & РИНЦ (Russia & $=0.156$ & PIF (India) & $=1.940$ \\
\hline & GIF (Australia) & $=0.564$ & ESJI (KZ) & $=8.716$ & IBI (India) & $=4.260$ \\
\hline & JIF & $=1.500$ & SJIF (Morocce & $=5.667$ & OAJI (USA) & $=0.350$ \\
\hline
\end{tabular}

скоростям, при которых деформация капель не имеет существенного значения.

Экстракция в период коалесценции капель. Скорость экстракции в этот период наименее изучена. Обнаружено, что количество вещества, переходящего из фазы в фазу в этот период, составляет 6-13\% от предельного, отвечающего состоянию равновесия. При этом количество вещества, переходящее из фазы в фазу, оказалось примерно одинаковыми в периоды образования и коалесценции капель. Для данного периода предложено, что основное сопротивление массопередачи при коалесценции сосредоточено в диспергируемой фазе и капли при осаждении мгновенно сливаются и образуют слой с равномерной первоначальной концентрацией вдоль сплошной поверхности, на которой происходит коалесценция. В этих случаях коэффициент массоотдачи можно определить по уравнению (6), которое может быть использовано c учетом величины сплошной поверхности коалесценции.

Уравнение (11) следует применять с осторожностью, так как оно не учитывает сопротивление массопередачи в сплошной фазе, которое во многих случаях может быть определяющим. Действительно, если слой сплошной фазы, примыкающий к поверхности коалесценции, недостаточно хорошо перемешан, то сопротивление в этой фазе будет контролировать процесс массопередачи. Полученные ими уравнения для расчета скорости процесса не основывались на конкретных физических моделях и не нашли широкого применения. Выдвинули предположение о чисто диффузионном механизме массопередачи при спокойном всплывании диспергированной фазы [5,c.1-12]. Однако, при экстракции в единичную каплю чисто диффузионный механизм массопередачи дает заниженные результаты. В работе [6, 843-862] предложили для расчета коэффициентов массопередачи в распылительной колонне уравнение, близкое к формуле Хигби [15]

$$
\kappa_{\mathrm{c}}=1,13 \sqrt{\frac{D_{\ni \phi}}{t_{0}}}
$$

где $D_{\text {эф }}$ - эффективный коэффициент диффузии, равной сумме коэффициентов молекулярной и турбулентной диффузии; $t_{0}$-период обновления поверхности.

Период обновления поверхности рассчитывают по формуле

$$
t_{0}=\frac{R}{U_{k}}
$$

где $R$ - радиус капли;

$$
U_{k} \text {-скорость капли }
$$

Как было показано выше, при экстракции единичными каплями, коэффициент массопередачи может быть определено по формуле
(11), если скорость процесса лимитируется массопередачей в сплошной фазе. Процесс массопередачи в распылительной колонне отличается от массопередачи экстракции единичными каплями, наличием стесненного потока диспергированной фазы.

Однако, детальное изучение этого процесса даёт основание предположить возможность применения указанных моделей для расчета коэффициентов массопередачи при экстракции в распылительной колонне.

Эксперименты показали, что коэффициенты массопередачи, рассчитанные по формуле Хигби для случая, когда лимитирующим является сопротивление сплошной фазы или по формуле Пилча и Эрдмана [7,c.3133-3164], когда лимитирующим является сопротивление диспергированной фазы.

Перенос массы внутри капли может принципиально осуществляться путем молекулярной диффузии или путем конвективного обмена. При этом простейшей моделью является модель, предполагающая массопередачу только путем молекулярной диффузии. Молекулярная диффузия играет значительную роль лишь в очень малых каплях и при малых скоростях относительного движения фаз. Диффузионная модель как бы ограничивает нижний предел, указывая наименьшую возможную степень насыщения. Согласно диффузионной модели, внутри капли отсутствует какое-либо конвективное движение, что, конечно, не соответствует действительности, ибо относительное движение фаз должно вызывать некоторое перемешивание внутри капли. Циркуляция внутри капель наблюдалась визуально рядом авторов [8.c.157-168] при добавлении в диспергированную фазу красителя. Установлено, что наличие циркуляции в каплях наблюдается при диаметре капли

$$
d>d_{\text {кр }}=2\left(\frac{\sigma}{\Delta \rho g}\right)^{0,5}(13)
$$

Массопередача при экстракции обычно происходит между системой капель (дисперсная фаза) и другой жидкостью (сплошная фаза). При этом дисперсная фаза движется через сплошную фазу. Для определения коэффициентов массопередачи нами предложено уравнение, учитывающее все физические параметры, участвующие в процессе массообмена в процессе экстракции [9,с.183-196]

$$
k_{i}=\left(\frac{1}{\beta_{i, C}}+\frac{m_{i}}{\beta_{i, D}}\right)
$$

где $m_{i}$-констант фазового равновесия компонентов, определяется из уравнения равновесия;

$\beta_{i, C}$ и $\beta_{i, D}$-коэффициенты массоотдачи компонентов в сплошной и дисперсной фазах. Коэффициент массоотдачи, если принять 


\begin{tabular}{|c|c|c|c|c|c|c|}
\hline \multirow{4}{*}{ Impact Factor: } & ISRA (India) & $=3.117$ & SIS (USA) & $=0.912$ & ICV (Poland) & $=6.630$ \\
\hline & ISI (Dubai, UAE & $=0.829$ & РИНЦ (Russia & $=0.156$ & PIF (India) & $=1.940$ \\
\hline & GIF (Australia) & $=0.564$ & ESJI (KZ) & $=8.716$ & IBI (India) & $=4.260$ \\
\hline & JIF & $=1.500$ & SJIF (Morocce & $=5.667$ & OAJI (USA) & $=0.350$ \\
\hline
\end{tabular}

движущую силу пропорциональной разности концентраций, можно определить по уравнению [10,c.428-435]

$$
\begin{gathered}
\beta_{i, D}=\frac{2 \pi^{2} D_{i, D}}{3 d_{k}}(15) \\
\beta_{i, \mathrm{C}}=\frac{2 \pi^{2} D_{i, C}}{3 d_{k}}(16)
\end{gathered}
$$

где $D_{i, D} \quad$ и $D_{i, C}$-коэффициенты молекулярной диффузии компонентов в дисперсной и сплошной фазах, соответственно. Они определяются по формулам

$$
\begin{aligned}
D_{i, D} & =\frac{7,4 \cdot 10^{-8}\left(\varphi_{D} \cdot M_{D}\right)^{0,5} \cdot T}{\mu_{D} \cdot V_{i}^{0,6}} \\
D_{i, C} & =\frac{7,4 \cdot 10^{-8}\left(\varphi_{C} \cdot M_{C}\right)^{0,5} \cdot T}{\mu_{C} \cdot V_{i}^{0,6}}
\end{aligned}
$$

где $\varphi_{D}$ и $\varphi_{C^{-}}$факторы ассосаций в дисперсной и сплошной фазах, соответственно $[11, \mathrm{c.50-55}$, 12,c.47-52].

$T$ - абсолютная температура процесса;

$M_{D}$ и $M_{C}-$ молекулярные массы дисперсной и сплошной фаз, соответственно;

$V_{i}$-мольный объем компонентов.

C помощью вышеуказанных формул определены коэффициенты молекулярной диффузии, массоотдачи, массопередачи для таких загрязнителей, как бутилгликоль,

\begin{tabular}{|c|c|c|c|c|c|c|}
\hline \multirow[t]{2}{*}{ Компоненты } & \multicolumn{2}{|c|}{$\begin{array}{l}\text { Коэффициенты } \\
\text { молекулярной } \\
\text { диффузии, м²/сек }\end{array}$} & \multicolumn{2}{|c|}{$\begin{array}{l}\text { Коэффициенты } \\
\text { массопередачи, } \\
\text { м/сек }\end{array}$} & \multirow{2}{*}{$\begin{array}{l}\text { Коэф- } \\
\text { фициенты } \\
\text { массо- } \\
\text { отдачи, } \\
\text { м/сек } K_{i} \text {. } \\
10^{4}\end{array}$} & \multirow{2}{*}{$\begin{array}{l}\text { Коэф- } \\
\text { фициенты } \\
\text { распреде- } \\
\text { ления }\end{array}$} \\
\hline & $\begin{array}{l}\text { дисперсная } \\
\text { фаза } \\
D_{i}^{D} \cdot 10^{9}\end{array}$ & $\begin{array}{l}\text { сплошная } \\
\text { фаза } \\
D_{i}^{C} \cdot 10^{9}\end{array}$ & $\begin{array}{l}\text { дисперсная } \\
\text { фаза } \\
K_{i}^{D} \cdot 10^{3}\end{array}$ & $\begin{array}{l}\text { сплошная } \\
\text { фаза } \\
K_{i}^{D} \cdot 10^{6}\end{array}$ & & \\
\hline Бутилгликоль & 4,18 & 0,894 & 0,113 & 0,242 & 0,231 & 0,2141 \\
\hline Фенилизопропанол & 3,07 & 0,658 & 0,083 & 0,108 & 0,170 & 0,2139 \\
\hline Уксусная кислота & 5,98 & 1,280 & 0,162 & 0,347 & 0,332 & 0,2142 \\
\hline
\end{tabular}
фенилизопропанол и уксусная кислота. Полученные результаты показаны в табл .2

Таблица 2. Коэффициенты молекулярной диффузии, массопередачи, массоотдачи и распределения

\section{Bblвodbl}

При помощи экспериментов получены данные для определения коэффициентов массопередачи в случае единичных капель.

Предложено, что количество компонента экстрагируемого за время контакта между фазами можно разделить на три части. Вещество в период: образования, падения и коалесценции капель.
Предложены механизмы процессов образования, падения и коалесценции капель.

Предложены уравнения для расчета коэффициентов массопередачи в период образования, падения и коалесценции капель.

По предложенной нами формуле рассчитаны коэффициенты молекулярной диффузии, массопередачи и массоотдачи.

\section{References:}

1. Evans, G. M., Jameson, G. J., \& Atkinson, B. W. (1992). Prediction of bubble size generated by a plunging liquids jet bubble column. Chem. Eng. Sci. 1992. v. 47, pp. 3265-3281.

2. Bin, A. K. (1993). Gas entrainment by plunging liquid jets. Chem. Eng. Sci. 1993, v. 48, pp. 3585-3596.

3. Sleicher, C. A. (2004). Maximum stable drop size in turbulent flow. AIChE Journal, 2004, v. 8, № 4, pp.471-486.

4. Kelbaliev, G. I., Suleymanov, G. Z., Fariborz, A., Gasanov, A. A., \& Rustamova, A. I. (2011). Ekstraktsionnoe razdelenie i ochitka stochnykh vod organicheskimi rastvoritelyami.
Khimicheskaya promyshlennost', 88, № 1, pp. 35-41.

5. Tobin, T., Muralidhar, R., Wright, H., \& Ramkrishna, D. (2010). Determination of coalescence frequencies in liquid-liquid dispersion: effect of drop size dependence. Chem. Eng. Sci., v.7, pp. 1-12.

6. Simmons, M. J. H., \& Azzopardi, B. J. (2001). Drop size distribution in dispersed liquid-liquid pipe flow. International Journal of Multiphase Flow, 2001, v. 27, pp. 843-862.

7. Angeli, P., \& Hewitt, O. F. (2000). Drop size distribution in horizontal oil-water dispersed flow. Chem. Eng. Sci., v. 55, pp. 3133-3164. 


\begin{tabular}{llllll} 
& ISRA (India) $=\mathbf{3 . 1 1 7}$ & SIS (USA) $=\mathbf{0 . 9 1 2}$ & ICV (Poland) & $\mathbf{= 6 . 6 3 0}$ \\
Impact Factor: & ISI (Dubai, UAE) $=\mathbf{0 . 8 2 9}$ & PUHЦ (Russia) $=\mathbf{0 . 1 5 6}$ & PIF (India) & $=\mathbf{1 . 9 4 0}$ \\
& GIF (Australia) $=\mathbf{0 . 5 6 4}$ & ESJI (KZ) & $\mathbf{8 . 7 1 6}$ & IBI (India) & $=\mathbf{4 . 2 6 0}$ \\
& JIF & $\mathbf{1 . 5 0 0}$ & SJIF (Morocco) $=\mathbf{5 . 6 6 7}$ & OAJI (USA) & $\mathbf{0 . 3 5 0}$ \\
\hline
\end{tabular}

8. Martinez-Bazan, C., Montanes, J. I., \& Lasheras, J. C. (1999). On the break-up of air bubble injected into fully developed turbulent flow. Part 1: Break-up frequency. Journal of Fluid Mechanics, 1999, v. 401, pp. 157-168.

9. Martinez-Bazan, C., Montanes, J. I., \& Lasheras, J. C. (1999). On the break-up of air bubble injected into fully developed turbulent flow. Part 2: Size population density. Journal of Fluid Mechanics, 1999, v. 401, pp. 183-196.

10. Kostoglou, M., \& Karabelas, A. J. (2001). A Contribution towards predicting the evolution of droplet size distribution in flowing dilute liquid/liquid dispersions. Chem. Eng. Sci., 2001, v. 56 , № 14 , pp. $428-435$

11. Gasanov, A. A. (2015). The mathematical description of liquid phase extraction of the industrial waste waters in cascaded mixing machines. Journal of Scientific Research and Development, 2(9), 50-55.

12. Gasanov, A. A., Suleymanov, G. Z., \& Aliev, A. M. (2014). Matematicheskoe modelirovanie protsessa zhidkostnoy ekstraktsionnoy ochistki mnogokomponentnykh sistem $\mathrm{v}$ raspylitel'noy ekstraktsionnoy kolonne. Zhurnal Zashchita okruzhayushchey sredy $v$ neftegazovom komplekse, № 8, pp. 47-52. 